\title{
STATISTICAL CONVERGENCE OF FUNCTIONAL SEQUENCES
}

\author{
B.T. BILALOV AND T.Y. NAZAROVA
}

\begin{abstract}
Statistical convergence in Lebesgue spaces is considered in this paper. A criterion for statistical convergence is given. It is shown that the known Tauberian theorems for scalar case are valid in this case, too.
\end{abstract}

1. Introduction. Apparently, the concept of statistical convergence of a sequence of numbers, as the generalization of the classical concept of a limit of a sequence, was first introduced by Fast in [5]. In $[\mathbf{6}, \mathbf{7}, \mathbf{1 6}$, 17], the basic properties of statistically convergent sequences have been studied. Later a lot of research appeared with various generalizations of this concept (see $[1,2,3,4,8,9,10,11,12,13,14,15,18]$ ). In $[\mathbf{1 0}, \mathbf{1 1}, \mathbf{1 2}]$, this concept was used in the theory of approximation by positive operators.

In this paper, we consider the statistical convergence in Lebesgue spaces $L_{p}$. We introduce the concept of statistical fundamentality in $L_{p}$ and prove its equivalence to the one of statistical convergence. We also prove that the Tauberian theorems of [6] stay valid in our case.

2. Preliminaries. We will use the standard notation. $\mathbb{N}$ will be the set of all positive integers; $\mathbb{R}$ is the set of all real numbers; $\chi_{M}(\cdot)$ is the characteristic function of $M$. Throughout this paper, we will denote by $|M|$ the cardinality of the set $M . C(M)$ denotes the space of continuous functions on $M$.

Recall the definition of the concept of statistical convergence of sequences of numbers. Let $\left\{a_{n}\right\}_{n \in \mathbb{N}} \subset \mathbb{R}$ be some sequence and $a \in \mathbb{R}$

2010 AMS Mathematics subject classification. Primary 11B05, 26A05, 40 A05.

Keywords and phrases. Statistical convergence, Lebesgue space, Tauberian theorems.

This work was supported by the Research Program Competition launched by the National Academy of Sciences of Azerbaijan (Program: Frame Theory Applications of Wavelet Analysis to Signal Processing in Seismology and Other Fields).

Received by the editors on September 24, 2013.

DOI:10.1216/RMJ-2015-45-5-1413 Copyright (C)2015 Rocky Mountain Mathematics Consortium 
a number. For all $\varepsilon>0$, put

$$
a^{\varepsilon} \equiv\left\{n \in \mathbb{N}:\left|a_{n}-a\right| \geq \varepsilon\right\} .
$$

The value

$$
\delta(M)=\lim _{n \rightarrow \infty} \frac{\sum_{k=1}^{n} \chi_{M}(k)}{n},
$$

is called a statistical density (stat density) of $M$.

Definition 2.1. The sequence $\left\{a_{n}\right\}_{n \in \mathbb{N}}$ is called statistically convergent to $a$, if $\delta\left(a^{\varepsilon}\right)=0$, for all $\varepsilon>0$, and this is denoted as

$$
\text { st } \lim _{n \rightarrow \infty} a_{n}=a \text {. }
$$

Many properties of statistically convergent sequences have been studied, and this concept is generalized in various directions. More details about these and related facts can be found in [1]-[18].

We will need the Tauberian theorems on statistically convergent sequences. Let $\left\{a_{n}\right\}_{n \in \mathbb{N}} \subset \mathbb{R}$ be a sequence. Let $\Delta a_{n}=a_{n}-a_{n+1}$. The following theorem is true.

Theorem 2.2. ([6]). Let $\mathrm{st} \lim _{n \rightarrow \infty} a_{n}=a$ and $\Delta a_{n}=\overline{\bar{o}}(1 / n)$. Then there exist $\lim _{n \rightarrow \infty} a_{n}$ and $\lim _{n \rightarrow \infty} a_{n}=a$.

The converse statement is not always true, i.e., the following theorem is true.

Theorem 2.3. ([6]). Let $\left\{r_{k}\right\}_{k \in \mathbb{N}}$ be a decreasing sequence of positive numbers such that $\left\{k r_{k}\right\}_{k \in \mathbb{N}}$ is unbounded. Then there exist $\left\{a_{k}\right\}_{k \in \mathbb{N}} \subset$ $\mathbb{R}:$ st $\lim _{k \rightarrow \infty} a_{k}=0$ and $\Delta a_{k}=\overline{\bar{o}}\left(r_{k}\right)$, while $\lim _{k \rightarrow \infty} a_{k}$ does not exist.

3. Statistical convergence in $L_{p}$. Let $\left\{f_{n}(x)\right\}_{n \in \mathbb{N}}$ be some sequence of functions $f_{n}: M \rightarrow \mathbb{R}$ and $M \subset \mathbb{R}$ some set. This sequence is called statistically convergent to $A$ at the point $x_{0} \in M$, if the sequence $\left\{f_{n}\left(x_{0}\right)\right\}_{n \in \mathbb{N}}$ statistically converges to $A$, i.e., st $\lim _{n \rightarrow \infty} f_{n}\left(x_{0}\right)=A$. This sequence is called statistically convergent to $f(x)$ on $M$ if

$$
\text { st } \lim _{n \rightarrow \infty} f_{n}(x)=f(x), \quad \text { for all } x \in M \text {. }
$$


Definition 3.1. We say that $\left\{f_{n}\right\}_{n \in \mathbb{N}}$ statistically uniformly converges to $f$ on $M$ if

$$
\text { st } \lim _{n \rightarrow \infty} \sup _{x \in M}\left|f_{n}(x)-f(x)\right|=0 \text {. }
$$

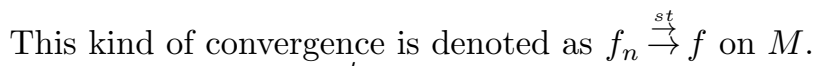

It is clear that, if $f_{n} \stackrel{s t}{\rightrightarrows} f$ on $M$, then the relation (3.1) holds. But the converse of this statement is not true.

Suppose that the relation (3.2) is true. Put $\alpha_{n}=\sup _{x \in M} \mid f_{n}(x)-$ $f(x) \mid$, for all $n \in \mathbb{N}$. Consequently, st $\lim _{n \rightarrow \infty} \alpha_{n}=0$. It is known that $\exists K \equiv\left\{n_{k}\right\}_{k \in \mathbb{N}}: n_{1}<n_{2}<\cdots, \delta(K)=1$ and $\lim _{k \rightarrow \infty} \alpha_{n_{k}}=0$. Thus, the sequence $\left\{f_{n_{k}}\right\}_{k \in \mathbb{N}}$ converges uniformly to $f$ in $M$. Hence, if $f_{n}(x)$ is continuous on $M$ and $f_{n} \stackrel{\text { 杰 }}{\rightarrow} f$ on $M$, then $f$ is also continuous on $M$. Moreover, for $M \equiv[a, b]$, we have

$$
\text { st } \lim _{n \rightarrow \infty} \int_{a}^{b} f_{n}(x) d x=\int_{a}^{b} f(x) d x .
$$

So, the following statement is true.

Statement 3.2. Let $\left\{f_{n}\right\}_{n \in \mathbb{N}} \subset C[a, b]$ and $f_{n} \stackrel{s t}{\rightrightarrows} f$ on $[a, b]$. Then $f \in C[a, b]$ and

$$
\text { st } \lim _{n \rightarrow \infty} \int_{a}^{b} f_{n}(x) d x=\int_{a}^{b} f(x) d x .
$$

Consider the $L_{p}$-case. Let $f_{n}, f \in L_{p}(a, b), 1 \leq p<+\infty$.

Definition 3.3. We say that $f_{n} \stackrel{s t}{\rightarrow} f$ in $L_{p}$ if

$$
\text { st } \lim _{n \rightarrow \infty} \int_{a}^{b}\left|f_{n}(x)-f(x)\right|^{p} d x=0, \quad 1 \leq p<+\infty .
$$

If the relation (3.3) is true, then there exists $K \equiv\left\{n_{k}\right\}_{k \in \mathbb{N}}, n_{1}<$ $n_{2}<\cdots, \delta(K)=1$ :

$$
\lim _{n \rightarrow \infty} \int_{a}^{b}\left|f_{n_{k}}(x)-f(x)\right|^{p} d x=0 .
$$


This implies that there exists a subsequence $\left\{f_{k_{n}}\right\}_{n \in \mathbb{N}}$ of the sequence $\left\{f_{n_{k}}\right\}_{k \in \mathbb{N}}$ such that $f_{k_{n}}(x) \rightarrow f(x)$ for almost every $x \in[a, b]$. Let

$$
\mathscr{K} \equiv\{K \subset \mathbb{N}: \delta(K)=1\} .
$$

In the sequel, we will need the following easily provable:

Lemma 3.4. Let $K_{j} \in \mathscr{K}, j=1,2 \Rightarrow K_{1} \bigcap K_{2} \in \mathscr{K}$.

In fact, let $I_{n} \equiv\{1 ; \ldots ; n\}$. We have:

$$
K_{1} \bigcap K_{2}=\left(K_{1} \bigcup K_{2}\right) \backslash\left[\left(K_{2} \backslash K_{1}\right) \bigcup\left(K_{1} \backslash K_{2}\right)\right] .
$$

Consequently,

$$
\begin{aligned}
& K_{1} \bigcap K_{2} \bigcap I_{n} \\
& =\left[\left(K_{1} \bigcup K_{2}\right) \bigcap I_{n}\right] \backslash\left[\left(\left(K_{2} \backslash K_{1}\right) \bigcup\left(K_{1} \backslash K_{2}\right)\right) \bigcap I_{n}\right] .
\end{aligned}
$$

As

$$
\left(\left(K_{2} \backslash K_{1}\right) \bigcup\left(K_{1} \backslash K_{2}\right)\right) \bigcap I_{n}=\left(\left(K_{2} \backslash K_{1}\right) \bigcap I_{n}\right) \bigcup\left(\left(K_{1} \backslash K_{2}\right) \bigcap I_{n}\right),
$$

taking into account

$$
\begin{aligned}
\left(K_{2} \backslash K_{1}\right) \bigcap I_{n} \subset K_{1}^{c} \bigcap I_{n} & \Longrightarrow \frac{\left|\left(K_{2} \backslash K_{1}\right) \bigcap I_{n}\right|}{\left|I_{n}\right|} \leq \frac{\left|K_{1}^{c} \bigcap I_{n}\right|}{\left|I_{n}\right|} \longrightarrow 0, \\
n & \rightarrow \infty, \\
\frac{\left|\left(K_{1} \backslash K_{2}\right) \bigcap I_{n}\right|}{\left|I_{n}\right|} & \longrightarrow 0, \quad n \rightarrow \infty,
\end{aligned}
$$

we get

$$
\frac{\left|\left(\left(K_{2} \backslash K_{1}\right) \bigcup\left(K_{1} \backslash K_{2}\right)\right) \bigcap I_{n}\right|}{\left|I_{n}\right|} \longrightarrow 0, \quad n \rightarrow \infty .
$$

From $\left(K_{1} \bigcap I_{n}\right) \subset\left(K_{1} \bigcup K_{2}\right) \bigcap I_{n}$ and $K_{1} \in \mathscr{K}$, it follows that

$$
\frac{\left|\left(K_{1} \bigcup K_{2}\right) \bigcap I_{n}\right|}{\left|I_{n}\right|} \longrightarrow 1, \quad n \rightarrow \infty,
$$


and hence, $K_{1} \cup K_{2} \in \mathscr{K}$. Then, from (3.4), we directly obtain

$$
\begin{aligned}
\frac{\left|K_{1} \cap K_{2} \bigcap I_{n}\right|}{\left|I_{n}\right|}= & \frac{\left|\left(K_{1} \bigcup K_{2}\right) \bigcap I_{n}\right|}{\left|I_{n}\right|} \\
& -\frac{\left|\left(\left(K_{2} \backslash K_{1}\right) \bigcup\left(K_{1} \backslash K_{2}\right)\right) \bigcap I_{n}\right|}{\left|I_{n}\right|} \longrightarrow 1, \quad n \rightarrow \infty,
\end{aligned}
$$

i.e., $K_{1} \bigcap K_{2} \in \mathscr{K}$. Lemma 3.4 is proved.

Definition 3.5. We say that $\left\{f_{n}\right\}_{n \in \mathbb{N}}$ is statistically fundamental (stfundamental) in $L_{p}$ if, for all $\varepsilon>0$, there exists $n_{\varepsilon} \in \mathbb{N}: \delta\left(\Delta_{\varepsilon}\right)=0$, where

$$
\begin{aligned}
\Delta_{\varepsilon} & \equiv\left\{n \in \mathbb{N}:\left\|f_{n}-f_{n_{\varepsilon}}\right\|_{p} \geq \varepsilon\right\}, \\
\|f\|_{p} & =\left(\int_{a}^{b}|f(t)|^{p} d t\right)^{1 / p} .
\end{aligned}
$$

It is absolutely clear that, if $f_{n} \stackrel{s t}{\rightarrow} f$ in $L_{p}$, then the sequence $\left\{f_{n}\right\}_{n \in \mathbb{N}}$ is st-fundamental in $L_{p}$. In fact, let $\varepsilon>0$ be an arbitrary number. Put $A_{\varepsilon} \equiv\left\{n:\left\|f_{n}-f\right\|_{p} \geq \varepsilon\right\}$. It is clear that $\delta\left(A_{\varepsilon / 2}^{c}\right)=1$, where $M^{c} \equiv \mathbb{N} \backslash M$. Take for all $n_{\varepsilon} \in A_{\varepsilon / 2}^{c}:\left\|f_{n_{\varepsilon}}-f\right\|<\varepsilon / 2$. We have

$$
\left\{n:\left\|f_{n}-f\right\|<\varepsilon / 2\right\} \subset\left\{n:\left\|f_{n}-f_{n_{\varepsilon}}\right\|<\varepsilon\right\},
$$

i.e., $A_{\varepsilon / 2}^{c} \subset A_{\varepsilon}^{c}$. Hence, $\delta\left(\Delta_{\varepsilon}^{c}\right)=1 \Rightarrow \delta\left(\Delta_{\varepsilon}\right)=0$.

Now, vice versa, let $\left\{f_{n}\right\}_{n \in \mathbb{N}}$ be st-fundamental in $L_{p}$. Denote by $O_{r}\left(x_{0}\right)$ the ball in $L_{p}$, i.e., $O_{r}\left(x_{0}\right) \equiv\left\{x \in L_{p}:\left\|x-x_{0}\right\|<r\right\}$. From stfundamentality, it follows that there exists $n_{j} \in \mathbb{N}: \delta\left(K_{j}\right)=1$, where $K_{j} \equiv\left\{n:\left\|f_{n}-f_{n_{j}}\right\|_{p} \leq 2^{1-j}\right\}, j=1,2$. By Lemma 3.4, we obtain $K_{1} \cap K_{2} \in \mathscr{K}$. Put $M_{1} \equiv \overline{O_{1}\left(f_{n_{1}}\right)} \bigcap \overline{O_{2^{-1}}\left(f_{n_{2}}\right)}(\bar{M}$ is a closure of $M$ in $\left.L_{p}\right)$. It is obvious that $f_{n} \in M_{1}$, for all $n \in\left(K_{1} \cap K_{2}\right) \equiv K_{(1)}$. Thus, there exists $n_{3} \in \mathbb{N}: K_{3} \in \mathscr{K}$, where $K_{3} \equiv\left\{n:\left\|f_{n}-f_{n_{3}}\right\|_{p} \leq\right.$ $\left.2^{-2}\right\}$. Let $K_{(2)}=K_{(1)} \bigcap K_{3}$. It is clear that $K_{(2)} \in \mathscr{K}$. Now, let $M_{2} \equiv M_{1} \cap \overline{O_{2^{-2}}\left(f_{n_{3}}\right)}$. Denote by $d_{p}(M)$ the diameter of the set $M$, i.e., $d_{p}(M)=\sup _{x, y \in M}\|x-y\|_{p}$. Continuing in the same way, we obtain a sequence of closed sets $\left\{M_{n}\right\}_{n \in \mathbb{N}}$ in $L_{p}$, whose diameters tend to zero: $d_{p}\left(M_{n}\right) \leq 2^{-n+1} \rightarrow 0, n \rightarrow \infty$. Moreover, $K_{(n)} \in \mathscr{K}$, where $K_{(n)} \equiv\left\{n: f_{n} \in M_{n}\right\}$. It is absolutely clear that $M_{n} \supset M_{n+1} \supset \cdots$. 
Take for all $x_{n} \in M_{n}$. We have

$$
\left\|x_{n}-x_{n+p}\right\|_{p} \leq d_{p}\left(M_{n}\right) \longrightarrow 0, \quad n \rightarrow \infty, \text { for all } p \in \mathbb{N}
$$

Hence, the sequence $\left\{x_{n}\right\}_{n \in \mathbb{N}}$ is fundamental in $L_{p}$, and let $x_{n} \rightarrow f$, $n \rightarrow \infty$. It is absolutely clear that $f \in \bigcap_{n} M_{n}$, i.e., $\bigcap_{n} M_{n}$ is non-empty. From $d_{p}\left(M_{n}\right) \rightarrow 0, n \rightarrow \infty$, it directly follows that $\{f\}=\bigcap_{n} M_{n}$, i.e., $\bigcap_{n} M_{n}$ consists of one element. As $K_{(m)} \in \mathscr{K}$, then there exists $\left\{n_{m}\right\}_{m \in \mathbb{N}} \subset \mathbb{N} ; n_{1}<n_{2}<\cdots$ :

$$
\frac{1}{n}\left|\left\{k \in I_{n}: k \in K_{(m)}^{c}\right\}\right|<\frac{1}{m}, \quad \text { for all } n>n_{m}
$$

Assume that

$$
\mathbb{N}_{0} \equiv\left\{k \in \mathbb{N}: n_{m}<k \leq n_{m+1} \quad \text { and } \quad k \in K_{(m)}^{c}\right\}
$$

and

$$
g_{k}= \begin{cases}f, & \text { if } k \in \mathbb{N}_{0} \text { and }\left(k>n_{1}\right) \\ f_{k}, & \text { if otherwise }\end{cases}
$$

Take $\varepsilon>0$. If $k \in \mathbb{N}_{0}$ and $\left(k>n_{1}\right)$, then $\left\|g_{k}-f\right\|_{p}=0<\varepsilon$. If $k \notin \mathbb{N}_{0} \Rightarrow k \in K_{(m)} \Rightarrow f_{k} \in M_{m} \Rightarrow\left\|f_{k}-f\right\|_{p} \leq \| f_{k}-$ $f_{n_{m}}\left\|_{p}+\right\| f_{n_{m}}-f \|_{p} \leq 2^{-m+2}<\varepsilon$, for sufficiently great values of $m$. Consequently, $\lim _{k \rightarrow \infty} g_{k}=f$. Let us show that $\delta(\widetilde{K})=0$, where $\widetilde{K} \equiv\left\{k \in \mathbb{N}: f_{k} \neq g_{k}\right\}$. Let $n_{m}<n<n_{m+1}$. Let us prove that

$$
\left\{k \leq n: f_{k} \neq g_{k}\right\} \subset\left\{k \leq n: k \in K_{(m)}^{c}\right\} .
$$

Let $f_{k} \neq g_{k}, k \leq n$. Consequently, $k \in \mathbb{N}_{0} \Rightarrow k \in K_{(m)}^{c}$. Thus,

$$
\frac{1}{n}\left|\left\{k \leq n: f_{k} \neq g_{k}\right\}\right| \leq \frac{1}{n}\left|\left\{k \leq n: k \in K_{(m)}^{c}\right\}\right|<\frac{1}{m} .
$$

It is clear that, if $n \rightarrow \infty$, then $m \rightarrow \infty$. Then, from the previous relation, we get

$$
\lim _{n \rightarrow \infty} \frac{\left|\left\{k \leq n: f_{k} \neq g_{k}\right\}\right|}{n}=0 .
$$

Consequently, $\left\{k \leq n: f_{k} \neq g_{k}\right\}^{c} \in \mathscr{K}$ and $\lim _{n \rightarrow \infty} g_{n}=f$. Let us 
show that $s t \lim _{n \rightarrow \infty} f_{n}=f$. Take $\varepsilon>0$. We have

$$
\begin{aligned}
\left\{k \leq n:\left\|f_{k}-f\right\|_{p} \geq \varepsilon\right\} \subset\left\{k \leq n: f_{k} \neq g_{k}\right\} & \\
& \bigcup\left\{k \leq n:\left\|g_{k}-f\right\|_{p} \geq \varepsilon\right\}
\end{aligned}
$$

As $\lim _{k \rightarrow \infty} g_{k}=f$ in $L_{p}$, then $\left\|g_{k}-f\right\|_{p}<\varepsilon$, for all $k \geq n_{\varepsilon}$. Consequently,

$$
\begin{aligned}
\left|\left\{k \leq n:\left\|g_{k}-f\right\|_{p} \geq \varepsilon\right\}\right| \leq n_{\varepsilon} \\
\quad \Longrightarrow \frac{1}{n}\left|\left\{k \leq n:\left\|g_{k}-f\right\|_{p} \geq \varepsilon\right\}\right| \rightarrow 0, \quad n \rightarrow \infty .
\end{aligned}
$$

Then, using (3.5), from (3.6) we obtain $1 / n\left\{k \leq n:\left\|f_{k}-f\right\|_{p} \geq \varepsilon\right\} \leq$ $1 / n\left\{k \leq n: f_{k} \neq g_{k}\right\}+1 / n\left\{k \leq n:\left\|g_{k}-f\right\|_{p} \geq \varepsilon\right\} \rightarrow 0, n \rightarrow \infty$. So, st $\lim _{n \rightarrow \infty} f_{n}=f$. Thus, we have proved the following theorem.

Theorem 3.6. Let $\left\{f_{n}\right\}_{n \in \mathbb{N}} \subset L_{p}$ be some sequence. Then the following statements are equivalent to each other:

(i) There exists st $\lim _{n \rightarrow \infty} f_{n}$;

(ii) $\left\{f_{n}\right\}_{n \in \mathbb{N}}$ is st-fundamental;

(iii) there exists $\left\{g_{n}\right\}_{n \in \mathbb{N}} \subset L_{p}$ : there exists $\lim _{n \rightarrow \infty} g_{n}$ and $\{n$ : $\left.f_{n}=g_{n}\right\} \in \mathscr{K}$.

This theorem immediately implies the following:

Corollary 3.7. Let $\left\{f_{n}\right\}_{n \in \mathbb{N}} \subset L_{p}$ and st $\lim _{n \rightarrow \infty} f_{n}=f$. Then there exists $\left\{n_{k}\right\}_{k \in \mathbb{N}} \subset \mathbb{N}: n_{1}<n_{2}<\cdots, \lim _{k \rightarrow \infty} f_{n_{k}}=f$ and $\delta\left(\left\{n_{k}\right\}_{k \in \mathbb{N}}\right)=1$.

In the case of a sequence of numbers, there is no regular matrix summation method which would include a statistical convergence (see [6]). The same statement stays valid in our case. The following lemma is true.

Lemma 3.8. Let $\left\{t_{k}\right\}_{k \in \mathbb{N}}$ be some sequence of numbers and $\sum_{k=1}^{\infty} \chi_{A_{0}}(k)$ $=+\infty$, where $A_{0} \equiv\left\{k \in \mathbb{N}: t_{k} \neq 0\right\}$. Then there exists $\left\{f_{k}\right\}_{k \in \mathbb{N}} \subset$ $L_{p}: \sum_{k=1}^{\infty} t_{k} f_{k}(t)=\infty$ for all $t \in[a, b]$. 
In fact, let's take a subsequence $\left\{m_{k}\right\}_{k \in \mathbb{N}}$ such that

$$
m_{k}>k^{2} \text { and } t_{m_{k}} \neq 0 \text {. }
$$

Define

$$
\begin{aligned}
x_{m_{k}}(t) & \equiv \frac{1}{t_{m_{k}}} \text { for all } t \in[a, b], \text { for all } k \in \mathbb{N} ; \\
x_{k}(t) & \equiv 0, \quad \text { for all } t \in[a, b], \text { for all } k \notin\left\{m_{1} ; m_{2} ; \ldots\right\} .
\end{aligned}
$$

We have

$$
\sum_{k=1}^{\infty} t_{k} x_{k}(t)=\sum_{k=1}^{\infty} t_{m_{k}} x_{m_{k}}(t)=\sum_{k=1}^{\infty} 1=\infty, \quad \text { for all } t \in[a, b] .
$$

On the other hand, it is easy to see that $x_{k} \stackrel{s t}{\rightarrow} 0$ in $L_{p}$. Lemma 3.8 is proved.

The following theorem is true.

Theorem 3.9. There is no matrix summation method which possesses statistical convergence in $L_{p}$.

Proof. From Lemma 3.8, it follows directly that if there is an appropriate matrix $A \equiv\left(a_{i j}\right)_{i, j \in \mathbb{N}}$, then for all $i \in \mathbb{N}$, there exists $m_{i} \in \mathbb{N}: a_{i j}=0$, for all $j \geq m_{i}$. It is absolutely clear that $A$ should have an infinite number of nonzero elements. Let $a_{n_{1} k_{1}^{\prime}} \neq 0$. Assume $k_{1}=\max \left\{j \in \mathbb{N}: a_{n_{1} j} \neq 0\right\}$. It is clear that $a_{n_{1} k_{1}} \neq 0$. Choose the indices $\left\{n_{m} ; k_{m}\right\}_{m \in \mathbb{N}}$ from the following conditions

$$
a_{n_{m} k_{m}} \neq 0, \quad k_{m} \geq m^{2} \quad \text { and } \quad a_{n_{m} j}=0, \text { for all } j>k_{m} .
$$

Now we define a sequence of functions $\left\{x_{n}(t)\right\}_{n \in \mathbb{N}}$ as follows:

$$
\begin{aligned}
x_{k_{1}}(t) & \equiv a_{n_{1} k_{1}}^{-1}, \quad \text { for all } t \in[a, b], \ldots, \\
x_{k_{m}}(t) & \equiv a_{n_{m} k_{m}}^{-1}\left[m-\sum_{i=1}^{m-1} a_{n_{i} k_{i}} x_{k_{i}}(t)\right], \quad \text { for all } t \in[a, b], \ldots, \\
x_{k}(t) & \equiv 0, \quad \text { for all } t \in[a, b], \text { for all } k \notin\left\{k_{1} ; k_{2} ; \ldots\right\}
\end{aligned}
$$

We have:

$$
y_{n}(t) \equiv(A \bar{x}(t))_{n}=\sum_{i: n_{i} \leq n} a_{n_{i} k_{i}} x_{k_{i}}(t)
$$


where $\bar{x}(t) \equiv\left(x_{1}(t) ; x_{2}(t) ; \ldots\right)$. Thus,

$$
y_{n} \equiv \sum_{i: n_{i} \leq n} 1, \quad \text { for all } n \in \mathbb{N} .
$$

In particular,

$$
y_{n_{m}}=m, \quad m \in \mathbb{N} \text {. }
$$

Obviously, $y_{n}(t) \stackrel{n}{\rightarrow} \infty$ in $L_{p}$. On the other hand, from $k_{m} \geq m^{2}$, it directly follows that $\left|\left\{k \leq n: x_{k} \neq 0\right\}\right| \leq \sqrt{n}$, and, as a result, st $\lim _{n \rightarrow \infty} x_{n}(t)=0$ in $L_{p}$.

Note that the examples in the scalar case, given in [6], show that the statistical convergence does not imply many summation methods. The same statement is true about the statistical convergence in $L_{p}$.

4. Tauberian theorems. Let $\left\{f_{n}\right\}_{n \in \mathbb{N}} \subset L_{p}$ be some sequence. Put $\Delta f_{n}=f_{n}-f_{n+1}$. The following analogue of Theorem 2.2 is true in $L_{p}$.

Theorem 4.1. Let st $\lim _{n \rightarrow \infty} f_{n}=f$ in $L_{p}$ and $\left\|\Delta f_{k}\right\|_{p}=\overline{\bar{o}}(1 / k)$. Then there exists $\lim _{n \rightarrow \infty} f_{n}$ in $L_{p}$ and $\lim _{n \rightarrow \infty} f_{n}=f$.

Proof. We will follow [6]. Assume st $\lim _{n \rightarrow \infty} f_{n}=f$. Then, by Theorem 2.3, there exists $\left\{g_{n}\right\}_{n \in \mathbb{N}} \subset L_{p}: \lim _{n \rightarrow \infty} g_{n}=f$ and $\left\{n: g_{n}=f_{n}\right\} \in \mathscr{K}$. Every $k \in \mathbb{N}$ can be represented as $k=m_{k}+p_{k}$, where

$$
m_{k}= \begin{cases}\max \left\{i \leq k: x_{i}=y_{i}\right\}, & A_{k} \neq \emptyset \\ -1, & A_{k}=\emptyset\end{cases}
$$

$A_{k}=\left\{i \leq k: x_{i}=y_{i}\right\}$. As proved in [6], it holds that

$$
\lim _{k \rightarrow \infty} \frac{p_{k}}{m_{k}}=0 \text {. }
$$

It is clear that there exists $B>0:\left\|\Delta f_{k}\right\|_{p} \leq B / k$, for all $k \in \mathbb{N}$. We have

$$
\begin{aligned}
\left\|g_{m_{k}}-f_{k}\right\|_{p} & =\left\|f_{m_{k}}-f_{m_{k}+p_{k}}\right\|_{p} \\
& \leq \sum_{i=m_{k}}^{m_{k}+p_{k}-1}\left\|\Delta f_{i}\right\|_{p} \leq B \frac{p_{k}}{m_{k}} \longrightarrow 0, \quad k \rightarrow \infty
\end{aligned}
$$


As $\lim _{k \rightarrow \infty} g_{m_{k}}=f$ in $L_{p}$, it directly follows that $\lim _{k \rightarrow \infty} f_{k}=f$.

The following analogue of Theorem 2.3 is also true.

Theorem 4.2. Let $\left\{r_{k}\right\}_{k \in \mathbb{N}}$ be a decreasing sequence of positive numbers such that $\left\{k r_{k}\right\}_{k \in \mathbb{N}}$ is unbounded. Then there exists $\left\{f_{k}\right\}_{k \in \mathbb{N}} \subset$ $L_{p}:$ st $\lim _{k \rightarrow \infty} f_{k}=0$ in $L_{p},\left\|\Delta f_{k}\right\|_{p}=\overline{\bar{o}}\left(r_{k}\right)$, while $\lim _{k \rightarrow \infty} f_{k}$ does not exist in $L_{p}$.

In fact, let $\left\{x_{k}\right\}_{k \in \mathbb{N}}$ be the sequence constructed in the proof of $[\mathbf{6}$, Theorem 4]. Let

$$
f_{k}(t)=x_{k}, \quad \text { for all } t \in[a, b], \text { for all } k \in \mathbb{N} .
$$

This is the sequence which was sought.

Note that the scheme of the proof of [6, Theorem 5] remains valid in this case, i.e., the following theorem is true.

Theorem 4.3. Let $\left\{k_{i}\right\} \subset \mathbb{N}$ be an increasing sequence satisfying

$$
\lim _{j \rightarrow \infty} \inf _{i \geq j} \frac{k_{i+1}}{k_{i}}>1,
$$

and $\left\{f_{k}\right\}_{k \in \mathbb{N}} \subset L_{p}: \Delta f_{k}=0$, if $k \neq k_{i}$, for all $i \in \mathbb{N}$. If there exists st $\lim _{k \rightarrow \infty} f_{k}=f$ in $L_{p}$, then there exists $\lim _{k \rightarrow \infty} f_{k}=f$ in $L_{p}$.

Remark 4.4. Similar results can be obtained for other functional spaces such as Sobolev spaces, Morrey-type spaces, etc.

\section{REFERENCES}

1. A. Basu and P.D. Srivastava, Statistical convergence on composite vector valued sequence space, J. Math. Appl. 29 (2007), 75-90.

2. J. Connor and K.-G. Grosse-Erdmann, Sequential definitions of continuity for real functions, Rocky Mountain J. Math. 33 (2003), 93-121.

3. J.S. Connor, The statistical and p-Cesaro convergence of sequences, Analysis 8 (1988), 47-63.

4. $\quad$ R-type summability methods, Cauchy criteria, p-sets, and statistical convergence, Proc. Amer. Math. Soc. 115 (1992), 319-327.

5. H. Fast, Sur la convergence statistique, Colloq. Math. 2 (1951), 241-244.

6. J.A. Fridy, On statistical convergence, Analysis 5 (1985), 301-313. 
7. J.A. Fridy, Statistical limit points, Proc. Amer. Math. Soc. 118 (1993), 11871192.

8. J.A. Fridy and M.K. Khan, Tauberian theorems via statistical convergence, J. Math. Anal. Appl. 228 (1998), 73-95.

9. J.A. Fridy and H.I. Miller, A matrix characterization of statistical convergence, Analysis 11 (1991), 59-66.

10. A.D. Gadjiev, Simultaneous statistical approximation of analytic functions and their derivatives by k-positive linear operators, Azerbaijan J. Math. 1 (2011), $57-66$.

11. A.D. Gadjiev and A.M. Ghorbanalizadeh, On the A-statistical approximation by sequence of $k$-positive linear operators, Proc. IMM NAS Azerb. 31 (2009), $41-51$.

12. A.D. Gadjiev and C. Orhan, Some approximation theorems via statistical convergence, Rocky Mountain J. Math. 32 (2002), 129-138.

13. M. Macaj and T. Salat, Statistical convergence of subsequence of a given sequence, Math. Bohem. 126 (2001), 191-208.

14. I.J. Maddox, Statistical convergence in a locally convex space, Math. Proc. Camb. Phil. Soc. 104 (1988), 141-145.

15. D. Rath and B.C. Tripathy, On statistically convergent and statistically Cauchy sequences, Indian J. Appl. Math. 25 (1994), 381-386.

16. T. Salat, On statistically convergent sequences of real numbers, Math. Slov. 30 (1980), 139-150.

17. I.J. Schoenberg, The integrability of certain functions and related summability methods, Amer. Math. Month. 66 (1959), 361-375.

18. A. Zygmund, Trigonometric series, Volume II, Cambridge University Press, London, 1979

B. Vahabzadeh 9, AZ1141, Institute of Mathematics and Mechanics of NAS of Azerbaijan

Email address: b_bilalov@mail.ru

B. Vahabzadeh 9, AZ1141, Institute of Mathematics and Mechanics of NAS of Azerbaijan

Email address: tubunazarova@mail.ru 